\title{
Meine Kinderjahre: Dichtung und Wahrheit
}

\author{
»Was? Wie? \\ 'ne Biographie? \\ Und Gott bewahre, \\ Bloß bis zum zwölften Jahre \\ Was man nicht alles erleben kann!« \\ Nehmen Sie's trotzdem freundlich an. \\ Fontane in ein Exemplar von Meine Kinder- \\ jahre für Otto Brahm
}

»Den Weg zurück ins Kinderland . . .« - so hat 1913 aphoristisch der Wiener Kulturkritiker und Satiriker Karl Kraus gesagt, der ja überhaupt von der Psychoanalyse behauptete, sie sei »jene Krankheit, für deren Therapie sie sich hält« - »Den Weg zurück ins Kinderland möchte ich, nach reiflicher Überlegung, doch lieber mit Jean Paul als mit S. Freud machen. « Oder mit Theodor Fontane? Denn einerseits hat Meine Kinderjahre noch ganz den unschuldigen Charme einer vor-Freudschen Autobiographie aus dem 19. Jahrhundert. Obwohl der Wiener Arzt Sigmund Freud seine revolutionierenden Entdeckungen über die kindliche Sexualität und ihren prägenden Einfluß auf das Erwachsenenleben des Menschen, die seine Zeitgenossen tief verstörten oder gar empörten, in den Jahren zu machen begann, als Fontanes Buch erschien, ist dieses von solchen Ungeheuerlichkeiten wie »Penisneid«, »anale Phase « und »Ödipuskomplex« frei. Es dauerte eine ganze Weile, bis sich die Psychoanalyse durchsetzte, und in der Kindheitsdarstellung war es noch ein weiter Weg von Meine Kinderjahre bis zu Jean-Paul Sartres Die Wörter.

Aber andererseits befriedigt Theodor Fontanes Selbstdarstellung auch den tiefenpsychologisch Interessierten, und schon einige der zeitgenössischen Rezensenten sahen in Meine Kinderjahre eine "sorgsame Seelenanalyse « das »Muster einer psychologischen Analyse ${ }^{2}{ }^{2}$ Das Buch bietet reiches Material für das Verständnis des Menschen und Autors Fontane und für die subtilen Beziehungen zwischen kindlichem Erlebnis und künstlerischem Schaffensprozeß.

Schon das Schreiben von Meine Kinderjahre hatte für Fontane offensichtlich eine psychotherapeutische Funktion. Die Beschäftigung mit seiner Kindheit und das Umsetzen dieser Erinnerungen ins literarische Werk halfen dem Dichter 1892 die schwerste psychosomatische Krise seines Alters zu 
überwinden. Sie wurde im März durch eine Influenza ausgelöst und brachte eine tiefgehende körperliche, seelische und geistige Ermattung mit sich. Die Lebenslust sank auf einen beängstigenden Tiefpunkt, die Inspiration versagte, die Arbeit an dem Roman Effi Briest, der 3 Jahre vorher begonnen worden war, stockte. Fontane wurde von Selbstzweifeln geplagt und gab sich seiner lang aufgestauten Verbitterung über die fortdauernde literarische Erfolglosigkeit hin, die künstlerisch lähmend und finanziell belastend wirkte. Diese Krise wurde verschärft durch Fontanes fixe Idee, er werde wie sein Vater mit 72 Jahren, also 1892, sterben:

Ein sonderbares Gefühl totalen Überflüssigseins beherrscht mich und wiewohl ich eigentlich nie »eine Zeit« gehabt habe, fühle ich doch, meine Zeit liegt zurück. ${ }^{3}$

In seiner tiefen Niedergeschlagenheit und seiner Schlaflosigkeit faßte Fontane sogar den Entschluß, Berlin aufzugeben und sich »nach Schmiedeberg für den Rest unserer Tage zurückzuziehen «. ${ }^{4}$ Den ganzen Sommer über, auch während des langen Urlaubs im Riesengebirge, klagte der 72jährige in seinen Briefen über eine »tiefe Müdigkeit«, einen »erbärmlichen Zustand «, »eine ganz gebrochene Kraft«, über »das Vereinsamen [ ... ] das immer wachsende Gefühl von der Lebensmühsal«, über »Trübsinn mit seinen grauen Schreckgespenstern«, über »Todesbangen [ ... ] Weltfremdheit [. . .] einen kraftlos halben Zustand ${ }^{5}{ }^{5}$ »Gehirn-Anämie « hatte eine »Breslauer Autorität, Prof. Hirt «, ${ }^{6}$ festgestellt, und Fontane unterzog sich bei ihm elektrischen Kuren. An schriftstellerische Tätigkeit war in diesem Zustand nicht zu denken; schon Briefe zu schreiben fiel ihm schwer. Aber am 1. November konnte Fontane seinem schlesischen Freund Georg Friedlaender unerwartet melden, »daß ich seit 8 oder 10 Tagen ins Schreiben gekommen bin, etwas das ich von mir gebrochenen Mann nicht erwartet hätte. Und zwar habe ich schon 4 Kapitel meiner Biographie (Abschnitt: Kinderjahre) geschrieben. Da mich dies Unterfangen sehr glücklich macht, so ist alle Correspondenz ins Stocken geraten ${ }^{?}{ }^{?}$

In rascher Folge wurden nun Meine Kinderjahre fertiggeschrieben. Weihnachten 1892 war die 1 . Niederschrift abgeschlossen, im April 1893 war der Band vollendet, Weihnachten 1893 lag er in den Buchhandlungen aus. Fontane hatte sich, wie er sagte, an diesem Buch wieder $»$ gesund geschrieben ${ }^{8}{ }^{8}$ Nicht die Breslauer Autorität, sondern der Hausarzt Dr. Delhaes erkannte den psychosomatischen Charakter der Krankheit Fontanes und bekämpfte sie dementsprechend nicht mit Medikamenten, sondern - auf Anregung von Familienmitgliedern des Dichters, wie man vermutet hat - mit einer Seelentherapie. Er empfahl dem alten Herrn, sich etwas völlig anderem zuzuwenden, und lenkte ihn durch den Hinweis auf die Kindheit von der bedrückenden Gegen- 
wart ab. Es erwies sich dann, daß diese Abwendung von der Gegenwart die erneute Zuwendung zu ihr künstlerisch um so fruchtbarer machte, denn was in der eigenen Kindheit Erlebnis gewesen war, gab nun für den Roman Effi Briest eine Fülle von Material her, das auf die Ebene des Symbols transponiert und damit zum Zeichen und Deutungsmedium im Handlungskontext wurde. Fontanes Kindheitswelt Swinemünde verwandelte sich in den Ort Kessin, wo Effi ihre ersten Ehejahre verbringt und den Ehebruch begeht, der ihr Leben zerstört. Es gehört ja zu den künstlerischen Eigenarten der Romane Fontanes, daß in Ihnen die seelischen Vorgänge in den Gestalten meist nicht direkt berichtet, sondern indirekt, durch die mit Bedeutung aufgeladene Wirklichkeit - Landschaft, Gegenstandswelt, Kunsterlebnisse usw. - gespiegelt und gedeutet werden; und gerade an der unterschiedlichen Verwendung desselben Rohmaterials in Selbstdarstellung und Roman läßt sich beobachten, wie die Transformation des Biographischen ins Dichterische sich vollzieht, wie Erfahrungen. Eindrücke und Erlebnisse des Autors sich in eine Symbolwelt verwandeln, die Seelisches enthält und enthüllt. Im Falle Fontanes handelt es sich dabei nicht um die einseitige Abhängigkeit des Romanhaften vom autobiographisch Gestalteten, sondern um wechselseitige Befruchtung, denn den Namen Bninski, der in Meine Kinderjahre auftaucht, hat Fontane schon 1878 in Vor dem Sturm verwendet, und schon in dem 1884 erschienenen Roman Graf Petöfy wird die Szenerie Swinemündes als Kindheitsort der Heldin von dieser selbst lebendig geschildert. Die Erinnerungen an seine Kindheit, die Fontane dafür aktiviert hat, sind sicher seiner späteren Autobiographie zugute gekommen. Auch Effi Briest war ja schon konzipiert, als Meine Kinderjahre geschrieben wurde, erfuhr aber erst danach seine eigentliche Ausarbeitung und gewann durch die Rückbesinnung Fontanes auf die Swinemünder Jahre in den Kessiner Partien an Dichte und geistig-seelischer Durchdringung.

Schon die Ähnlichkeit der Schauplätze ist unübersehbar, angefangen bei den Namen, die hier wie dort vorkommen: Thomsen/Thompson, die "Plantage « und die Oberförsterei von Uvagla/Pudagla; und Fontane selbst hat ja auch angemerkt, daß er »dem räthselhaften Kessin [. . .] die Scenerie von Swinemünde gegeben ${ }^{9}$ habe, wobei er die internationale Atmosphäre des Städtchens ins Geheimnisvoll-Unheimliche gesteigert hat.

$\mathrm{Da}$ ist etwa der Spuk in der väterlichen Apotheke. Angeblich geistert der verstorbene Vorgänger des Vaters auf dem Boden herum - »de oll Geisler geiht wedder üm« -, was die Einbildungskraft des "phantastischen Kindes« Fontane erregt und beschäftigt, das schon damals nach allem, was einen »et- 
was aparten und das nächtlich Schauerliche streifenden Charakter hatte, begierig verlangte«. Dieser Charakterzug des Kindes Fontane wird in Meine Kinderjahre immer wieder betont und läßt den zukünftigen Balladendichter und Romancier ahnen, für den der Sinn für das Besondere und die lebhafte Einbildungskraft konstitutiv sind, aber auch Effi Briest wird schon zu Anfang des Romans gerade mit den Wörtern »phantastisch « und »apart « gekennzeichnet, und später warnt sie ihr Mann: »Aber hüte dich vor dem Aparten oder was man so das Aparte nennt. Was dir so verlockend erscheint [...] das bezahlt man in der Regel mit seinem Glück. [...] Immer Phantasien, mal so, mal so.« (EB 87) In dem Roman gestaltet Fontane die Erinnerung an das Gespenst des toten Apothekers in dem spukenden Chinesen in Effis Kessiner Ehehaus zu einem, wie er selbst betont hat, »Drehpunkt für die ganze Geschichte« um, der auf immer neue Weise Effis Ängste und Schuldgefühle, die prekäre Beziehung zu ihrem Mann und das Verhältnis zu ihrem Liebhaber beleuchtet und das Buch durchzieht. Da ist der "getrocknete Buttfisch«, der bei der Ankunft der Fontanes in ihrer Apotheke in Swinemünde »von der gewölbten Decke« des Laboratoriums hängt, den 7 jährigen beeindruckt und zur Atmosphäre des »alchymistischen Raumes« beiträgt. Er hat sich in Effi Briest in ein ganzes Arsenal unheimlicher Dinge verwandelt, die die junge Ehefrau in Kessin im Hausflur empfangen:

Quer über den Flur fort liefen drei, die Flurdecke in ebenso viele Felder teilende Balken; an dem vordersten hing ein Schiff mit vollen Segeln, hohem Hinterdeck und Kanonenluken, während weiterhin ein riesiger Fisch in der Luft zu schwimmen schien. Effi nahm ihren Schirm, den sie noch in Händen hielt, und stieß leis an das Ungetüm an, so daß es sich in eine langsam schaukelnde Bewegung setzte.

»Was ist das, Geert?« fragte sie.

"Das ist ein Haifisch.«

»Und ganz dahinten das, was aussieht wie eine große Zigarre vor einem Tabaksladen?«

»Das ist ein junges Krokodil.«(EB 50)

So wird Effis neues Leben von Anfang an in eine Atmosphäre des Exotischen, Beängstigenden und Bedrohlichen getaucht. Sie lebt gewissermaßen nicht auf dem Land, sondern im Wasser, wie es ihrer seelischen Disposition entspricht.

Da ist im 13. Kapitel von Meine Kinderjahre der Scherz, daß Dr. Lau, dem Livius-Kenner, die Swinemünder Konsuln kaum imponiert haben können, woraus Fontane in Effi Briest einen Dialog macht, bei dem Effi erst Konsuln mit Liktoren verwechselt und dann nicht weiß, daß es moderne Handelskon- 
suln gibt, und so ihrem Mann gegenüber ihre Unbildung beweist - ein Aspekt der Inkompatibilität der Ehepartner.

$\mathrm{Da}$ ist das gefahrvolle und gerade darum so reizvolle Schaukeln, das Fontane im 4. Kapitel von Meine Kinderjahre beschreibt:

Schöner aber als alles das, war, für mich wenigstens, eine zwischen zwei Holzpfeilern angebrachte, ziemlich baufällige Schaukel. Der quer überliegende Balken fing schon an morsch zu werden und die Haken, an denen das Gestell hing, saßen nicht allzu fest mehr. Und doch konnt' ich gerade von dieser Stelle nicht los und und setzte meine Ehre darin, durch abwechselnd tiefes Kniebeugen und elastisches Wiederemporschnellen, die Schaukel derartig in Gang zu bringen, daß sie mit ihren senkrechten Seitenbalken zuletzt in eine fast horizontale Lage kam. Dabei quietschten die rostigen Haken und alles drohte zusammen zu brechen. Aber das gerade war die Lust, denn es erfüllte mich mit dem wonnigen und allein das Leben bedeutenden Gefühle: dich trägt dein Glück. (41 f.)

Diese Schaukel steht dann auch im Garten von Effi Briests Elternhaus, und so wird aus der eigenen Disposition des Autors ein Charakterzug seiner Romanheldin, der sie psychologisch deuten hilft:

Ich klettre lieber, und ich schaukle mich lieber, und am liebsten immer in der Furcht, daß es irgendwo reißen oder brechen und ich niederstürzen könnte. Den Kopf wird es ja nicht gleich kosten. (EB 34)

Oder:

Am liebsten aber hatte sie wie früher auf dem durch die Luft fliegenden Schaukelbrett gestanden und in dem Gefühle: >Jetzt stürz' ich<, etwas eigentümlich Prickelndes, einen Schauer süßer Gefahr empfunden. (EB 118)

$\mathrm{Da}$ dieses im Schaukeln greifbare Bedürfnis Effis, sich in Gefahr zu begeben und als »Tochter der Luft« (EB 8) den festen Boden unter den Füßen zu verlieren, auf ihre Bereitschaft hinweist, auch im Leben Risiken einzugehen, zeigt sich darin, daß sie sich »im Schaukelstuhl« wiegt, als nach ihrer eigenen späteren Erkenntnis ihre verbotene Beziehung zu ihrem Liebhaber beginnt, der gerade in dieser Szene vom Strand kommt. Er hat bei 9 Grad in der Ostsee gebadet; ihm also kann das Wasser nichts anhaben - Details, die in den Zusammenhang des gewichtigsten Motivs gehören, das Fontane aus eigenen Kindheitserlebnissen gespeist und in Meine Kinderjahre behandelt hat: des Wassermotivs und der Gefahren, die dieses Element für Effi hat, das zum 
zentralen Symbol ihrer Persönlichkeit wird. Schon daß sie ursprünglich Betty von Ottersund heißen sollte, deutet ihre verhängnisvolle Beziehung zum Meer an.

In Meine Kinderjahre empfängt den jungen Theodor bei der Ankunft in Swinemünde das immerwährende Rauschen der Ostsee, die er noch nicht kennt, und diese erste, noch indirekte Begegnung mit der See ist zugleich eine Beschwörung ihrer Gefahren. "Mitunner «, berichtet der alte Kutscher Ehm, »kümmt se bis ran un steiht hier rümm in alle Straten. Un so'n Lütting wie du, de kann denn versupen. " Noch mehrmals im Laufe des Buches wird von »Wasserfährlichkeiten « berichtet, in die der kleine Junge gerät, wie überhaupt der Charme Swinemündes von seiner Lage am Wasser herrührt. Aus diesen Erinnerungen Fontanes an die Ostsee ist das fruchtbarste Symbol seines gesamten Romanwerks hervorgegangen, das immer wieder gerade im Zusammenhang mit jungen Frauen und ihren sexuellen Gefährdungen verwendet wird. Auf Fontanes Melusinen - in Der Stechlin führt eine verführerische Frau sogar diesen Vornamen -, die zum Wasser in einer unerklärlichen, Erotisches andeutenden Beziehung stehen, ist öfter hingewiesen worden, aber nirgendwo wird das Motiv so durchgängig und aspektenreich auf eine Romanheldin bezogen wie in Effi Briest. Schon zu Beginn des Buches ist Effi »wie ein Schiffsjunge « (EB 15) gekleidet und begibt sich im Boot auf den Teich im väterlichen Garten, wobei sie mit ihren Freundinnen über ehebrecherische Frauen spricht. Sie wächst im »Luch « auf, einem Sumpfgebiet, das sie selbst ausdrücklich mit dem »Schloon« in der Nähe Kessins vergleicht, dem unterirdischen Rinnsal, das zeitweise zu einem reißenden Strom anwächst, in dem sie unmittelbar vor der Liebeserklärung ihres Liebhabers zu versinken droht und das auch dem Namen nach der realen Umgebung Swinemündes entstammt. Überhaupt übt das Meer auf Effi eine unwiderstehliche Anziehungskraft aus, so daß sie »am liebsten gleich in die Brandung « flöge, sich einbildet, »die Meerfrauen singen « (EB 157) zu hören, und von dem Scheitern eines Schiffes vor dem Hafen so ergriffen wird, daß sie in Tränen ausbricht. $\mathrm{Da}$ verwundert es nicht, daß ihr Liebhaber sie schon vor dem eigentlichen Ehebruch auf dem Umweg über Heinrich Heines Gedicht Das Seegespenst einsam und verloren in einer versunkenen Stadt sitzen sieht. Das Stichwort für das Gespräch über das Heinesche Gedicht aber bildet Vineta, die wendische Stadt, die angeblich vor der Insel Usedom in der Ostsee versunken ist, worauf Effi hinweist.

Und damit ist der Bogen zum Ursprung des ergiebigen Wassersymbols geschlagen, zu den Erlebnissen Fontanes als Kind, denn auf das geheimnisvolle heidnische Vineta wird in Meine Kinderjahre mehrmals hingewiesen.

Die Gefahren des Wassers waren es offenbar auch, die Fontane dazu bewogen, die junge protestantische Schauspielerin Franziska Franz in Graf Petöfy, deren Ehe mit einem alten katholisch-ungarischen Grafen zum Schei- 
tern verurteilt ist, ebenfalls in seiner eigenen Kindheitsstadt anzusiedeln. Sie stammt aus »einer kleinen Hafen- oder Badestadt an der Ausmündung der Oder« (GP 64), die deutlich die Züge Swinemündes trägt, wie man es aus Meine Kinderjahre kennt:

»Da haben wir zunächst unsern Strom, dessen breite Wasserfülle schon die Nähe des Meeres ahnen läßt. Und keine tausend Schritte vor seiner Mündung, da wächst die Stadt auf und zieht sich einreihig an einem Pfahlwerk entlang, an dessen steil abfallender Wasserseite die Schiffe liegen, groß und klein, mit ihren vergoldeten Namen am Spiegel und einer überlebensgroßen, in Holz geschnittenen Figur am Bug. Auf dem breiten Damm aber, der dem Schlängellaufe des Flusses folgt, bewegen sich Handel und Verkehr wie unter einem Walde spalierbildender Maste. Denn zu beiden Seiten erheben sich diese Maste, sowohl auf den Schiffen wie vor den Häusern gegenüber.«

"Und wie sind diese Häuser?«

"Oft so niedrig, daß man die Hand aufs Dach legen kann. Aber immer frisch geweißt. Und auf dem hohen Dache, das meist dreimal höher ist als das eigentliche Haus, auf diesem Dach erhebt sich ein Giebel und auf dem Giebel eine Flaggenstange, daran ein langes schmales Band oder auch eine sich bauschende Flagge weht. Und keine Flagge dieselbe; denn in jedem dieser Häuser hat ein anderes Land seinen Sitz und seinen Schutz, und während über dem einen der österreichische Doppeladler flattert, flattert über dem andern der türkische Halbmond oder der chinesische Drache. Es gibt nichts Bunteres und Lachenderes als das Flaggen einer solchen Hafen- und Handelsstadt.« (GP 65 f.)

Es würde zu weit führen, hier darauf einzugehen, daß gerade Österreichisches, Türkisches und Chinesisches auch im Kessin von Effi Briest eine Rolle spielen. Franziska erzählt noch weiter vom Leben am Wasser und bezaubert damit den alten Grafen Petöfy, und ihre Erinnerungen an ihre Kindheit laufen auf die Schilderung einer Sturmnacht im November hinaus, bei der der Nordwestwind eine Überschwemmung hervorruft, was ja auch kurz vor Effis Ehebruch in Kessin geschieht:

Denn der Nordwester staute nicht nur den Strom zurück, sondern trieb auch das Flutwasser mit solcher Gewalt von draußen her in den Strom hinein, daß es am Kai hin oft nur noch zollbreit unter der obersten Balkenlage stand. Und einmal - ich seh' es, als ob es gestern gewesen wäre stieg es drüber hinaus, und im $\mathrm{Nu}$ war die niedriger liegende Stadt ein See von einem Punkte zum andern, und in unsern Flur hinein stürzte die 
Welle. Da schrien wir auf, denn nun erfüllte sich unser Schicksal, und wir mußten untergehen, wie Vineta untergegangen war. (GP 68)

Daß auch hier die Gefahr der Überschwemmung symbolisch auf Franziskas späteres Überwältigtwerden von ihrer Sexualität vorausdeutet, in der sich ihr Schicksal erfüllt, als ihr Mann ihren Ehebruch erfährt und Selbstmord begeht, ergibt sich aus dem wiederholten Auftauchen des Wasser-Motivs im Lauf der Handlung. So wird sich Franziska ihrer Einsamkeit, Sehnsucht und Frustration auf dem ungarischen Schloß ihres Mannes bewußt, als im Herbst ausdauernde Regenfälle reißende Bäche auf dem Schloßberg bilden, und so findet die entscheidende Begegnung mit ihrem Geliebten bei einer Fahrt über einen unberechenbaren See statt, wo ein durch einen plötzlichen Sturm hervorgerufener Strudel das Boot zu verschlingen droht.

Zwischen Autobiographie und Romanwerk laufen bei Fontane also vielfältige Fäden hin und her, so daß Anderson ${ }^{10}$ im Zusammenhang mit Meine Kinderjahre von der »Brücke zwischen Leben und Kunst « gesprochen hat. Hätte der Autor aber seine Swinemünder Kinderzeit nicht beschrieben, dann könnte der Fontane-Leser heute nicht verfolgen, wieviel an Menschen, Gegenständen und Episoden aus eigenem Erleben in seine Romane eingegangen ist. Das Charakteristische gerade des Fontaneschen Werks ist es dabei, daß die Elemente der Wirklichkeit - Erlebtes, Erfahrenes, Beobachtetes, Erlittenes - nicht plan in den Romantext übernommen werden, sondern daß sie eine aus Realität und Phantasie zusammengefügte Kunstwelt konstituieren helfen, die ästhetisch und symbolisch durchkomponiert ist. Wenn der Leser dabei dem Autor so häufig erst auf die Spur kommen muß, weil dieser die Bedeutung seiner Szenen gerade im scheinbar realen Detail verbirgt, dann trägt auch hier, worauf Anderson hingewiesen hat, Meine Kinderjahre zum Verständnis bei. Die Autobiographie ist ein Buch, in dem ein gut 70jähriger in der Kenntnis seines Lebenswegs als Mensch und Dichter seine eigene Zeit zwischen dem 7. und 12. Lebensjahr auch im Hinblick auf spätere Entwicklungen schildert und deutet. Es ist daher eine Darstellung seiner Ursprünge und ein Dokument künstlerischer Gestaltung, das eine Auswahl des Erlebten und eine Interpretation der Ereignisse darstellt, worauf Fontane im Untertitel »autobiographischer Roman « und auch im Vorwort hinweist, denn darin möchte er seine "Kindheitsgeschichte als eine Lebensgeschichte « gewertet wissen, weil - und hier liegt eine weitere psychologische Dimension des Buches - im frühen Menschen der ganze Mensch stecke; und der Autor hält die Beziehung zwischen dem Kind und dem Erwachsenen im Laufe des Buches auch ganz be- 
wußt lebendig, indem er immer wieder auf sein späteres Leben überblendet. Er spricht etwa davon, daß er möglicherweise »immer noch einen bescheidenen Bestand von Haar « habe, weil er als Kind »mit dem sogenannten engen Kamm« gequält wurde; daß er immer noch gern »den appetitlichen Qualm« von heißem Teer rieche, den er beim Kalfatern der Schiffe in Swinemünde zuerst eingeatmet habe; und daß er von klein auf an Zimmern vor allem das Gemütliche geschätzt habe. Er erinnert an seine Kriegsbücher und läßt sich dabei über schriftstellernde Fachleute und Laien auf verschiedenen Gebieten aus und blendet in dem berühmten 16. Kapitel ganz in eine spätere Zeit hinüber. Deutlich ist hier die enge Verwandtschaft von Alter und Kindheit zu spüren, für die Justinus Kerner in seinem Bilderbuch aus meiner Knabenzeit (1840) so verständnisvolle Worte gefunden hat:

Bilder und Erlebnisse in der Jugend gehen, je mehr wir uns von ihr entfernen, in um so hellerem Lichte in uns auf dem schwarzen Grunde des Alters auf; das Ende berührt den Anfang, wir nähern im Alter uns selbst wieder mehr der Kindheit. Es gab Greise, denen die Erinnerung aus ihrem Jünglings- und Mannesleben völlig verschwand, während die Zeit ihrer Kindheit ihnen wieder zur Gegenwart wurde, daß sie vermeinten, noch Kinder zu sein. ${ }^{11}$

Es ist daher berechtigt zu fragen, welche Wesenszüge dem alten Fontane an seinem Kindheitsporträt wichtig waren. Dabei ist schon auf seinen Sinn für das Aparte und das Abenteuerliche und auf seine Empfänglichkeit für das Unheimliche hingewiesen worden, für das die Wirkung des auf dem Dachboden aufbewahrten Rades, mit dem der Mörder Hannacher umgebracht wurde, und der tiefe Eindruck der Hinrichtung des Mörders Mohr wirkungsvolle Beispiele bilden. Sensibilität und Verletzlichkeit, Führungsbewußtsein und Eitelkeit sind andere Charakteranlagen, die Fontane im Rückblick wichtig erschienen, und eine besonders ist aufschlußreich für die spezifische Gestaltungstechnik des späteren Romanciers Fontane: das Bedürfnis, sich zu verstecken. Der Autor berichtet von seiner »Versteckspiel-Passion«, der er ratlos gegenübersteht. Dabei bestand für ihn das Glücksgefühl darin, stundenlang gesucht und nicht gefunden zu werden, so daß er sein Versteck im Triumph als Sieger verlassen konnte. Anderson hat in seinem Aufsatz die Leidenschaft des Kindes auf den alten Schriftsteller bezogen, der in seinen Romanen mit dem Leser auf andere Weise das Versteckspiel veranstaltet, das ihn einst als Kind so glücklich gemacht hat. Bei Graf Petöfy und Effi Briest gibt gerade die Autobiographie dem Leser einige Hinweise auf das, was an Bedeutung ins literarische Werk eingewoben ist. Aber auch die Neigung zum Versteckspiel selbst hat der Verfasser von sich auf seine Heldin Effi Briest übertragen, die, wie wir gesehen haben, so viele Fontanesche Züge bekommen hat, daß die zeitli- 
che Nähe der beiden Werke sich auch als geistige Nähe und psychische Verwandtschaft von Autor und Heldin herausgestellt hat. Effi versteckt sich schon im 2. Kapitel vor ihren Freundinnen - und zwar hinter Rhabarberstauden, deren Blätter sie mit Feigenblättern vergleicht - und freut sich, nicht gefunden zu werden. Nach dem Ehebruch betreibt sie dann das Versteckspiel in größerem Stil vor ihrem Mann und der Gesellschaft.

Wenn es um den späteren Romancier geht, muß im Zusammenhang mit Meine Kinderjahre vor allem noch auf 2 Dinge aufmerksam gemacht werden. Fontane spricht darin von seiner Empfänglichkeit »für die Zauber von Form und Farbe « und liefert an anderer Stelle Beispiele, wie Szenen sich ihm wegen ihres Farb- oder Formeindrucks besonders eingeprägt haben, so die Episode, in der seine Mutter nachts von einer Gesellschaft nach Hause kommt, weil sie ihre Kinder in Gefahr glaubt. Fontane hat davon vor allem den Gegensatz zwischen dem dunkelroten Brokatkleid und ihrem schwarzen Haar in Erinnerung behalten. Ähnlich sind es einige Seiten später »die schwarz- und schwefelgelb gestreifte Weste « auf dem Ölbild seines Großvaters und das abendliche Bild von roter Sonne, grünen Tannen, dunkler Wasserfläche und weißen Seerosen, das sich ihm nachdrücklich eingeprägt hat. Hier sind biographische Ursprünge der farbstarken Bilder zu greifen, die Fontane immer wieder in seinen Romanen stellt.

Das andere Element ist die Neigung zu esprithaft-frivolen Gesprächen mit Damen, von denen Fontane sagt, daß er »diese Neigung sogar in meine Schreibweise mit herübergenommen « habe. Der Zusatz, »wenn ich entsprechende Szenen in meinen Romanen und kleinen Erzählungen lese, so ist es mir mitunter, als hörte ich meinen Vater sprechen«, enthält einen Schlüssel zu seinem Selbstverständnis, denn der alte Schriftsteller läßt bei seiner Selbstcharakterisierung als Kind keinen Zweifel daran, daß er sich wesensmäßig seinem Vater viel verwandter empfindet als seiner Mutter, ja, man kann $\mathrm{Mei}$ ne Kinderjahre als eine Art später Liebeserklärung des Sohnes an seinen Vater Louis Henri Fontane lesen, den "großen stattlichen Gascogner voll Bonhomie, dabei Phantast und Humorist, Plauderer und Geschichtenerzähler «; und ein menschlicheres und einfühlsameres Porträt eines Vaters als Fontanes »Intermezzo « des Besuchs bei dem alten Herrn »vierzig Jahre später « ist sicher selten geschrieben worden. Während die strenge, rechtschaffene Mutter eher ein Schatten bleibt und noch das zweitletzte Kapitel mit dem fatalen Weihnachtsgeschenk von ihrer mangelnden Einfühlung in ihr Kind berichtet, ist die vitale, wenn auch haltlose Persönlichkeit des Vaters beinahe immer gegenwärtig, und der Leser kann sich des Eindrucks nicht erwehren, daß sich die Konstellation der elterlichen Ehe in gewisser Weise beim Sohn wiederholt hat und daß der Autor sich dessen in seiner Schilderung auch bewußt war. Das Recht mag auf der Seite der Frauen gewesen sein, aber Liebenswürdigkeit und Leben waren auf der der Männer - umgekehrt übrigens wie in Effi 
Briests Ehe. Es ist bezeichnend, daß Fontane 1870 in einem Brief an seine Frau, die mit seiner Entscheidung, seine Stellung bei der Kreuzzeitung aufzugeben, nicht einverstanden war und ihm Vorwürfe gemacht hatte, schrieb: "Meine Mutter freute sich auch immer, wenn mein Vater fror. ${ }^{12} \mathrm{Zu}$ beschönigen versucht der Sohn das unverantwortliche Verhalten seines.Vaters nicht, aber er schildert auch seine Schwächen so, daß die tiefe Sympathie und die seelische Verwandtschaft mit ihm spürbar bleiben.

Der Leser selbst mag die vielen Ähnlichkeiten zwischen Vater und Sohn entdecken, aber eine Beziehung, die Fontane nicht herstellt, muß erwähnt werden. Mehrmals gibt der Autor in Meine Kinderjahre Beispiele für die zwanglose, fesselnde, untheoretische, lebendige Art der Wissensvermittlung des Vaters, die dieser selbst seine "sokratische Methode « nennt und die er begeistert auch an dem neuen Lehrer des Sohns, Dr. Lau, feststellt. Fontane sagt es nicht, aber der Leser weiß: Er selbst war begnadet mit der "sokratischen Methode«. Die Wanderungen durch die Mark Brandenburg, die Kriegsund Reisebücher und nicht zuletzt die autobiographischen Schriften sind der Beweis. Das ist einer der Gründe, weshalb auch die >Sachbücher Fontanes anders als alles Vergleichbare seiner Zeit nicht veraltet sind, sondern auch nach 100 Jahren noch ihre Lesbarkeit bewahrt haben.

Die deutsche Autobiographie im modernen Sinn ist ihrem Wesen und ihrer historischen Entstehung nach mit dem Aufstieg des Bürgertums in der 2. Hälfte des 18. Jahrhunderts verbunden. Anders als für den Adel, der seinen Stolz und sein Selbstbewußtsein aus Familientraditionen bezog - je länger die Reihe der Ahnen, desto mehr Ansehen -, hing der Selbstwert des bürgerlichen Menschen von der eigenen Lebensleistung ab. Was er aus seinem Leben gemacht hatte, war sein Stolz. Dieser Gedanke hat ursprünglich eine religiöse Komponente. Nach dem Pietismus stand jede Seele in einer engen persönlichen Beziehung zu Gott; der Mensch schuldete diesem daher Rechenschaft, wie er mit dem ihm vertrauten Pfunde seines Lebens gewuchert hatte. Das förderte die Reflexion über das eigene Leben, das so wunderbar von Gott geleitet wurde, und das Bedürfnis, pädagogisch zu wirken und durch die Darstellung seiner Lebensfährnisse die Jugend vor falschen Wegen zu bewahren. In dieser Tradition entstand auch die Gewohnheit, das Leben als Wanderung oder Reise zu betrachten, die man zu einem erfolgreichen Ende führen muß ein Gedanke, der metaphorisch noch das ganze 19. Jahrhundert durchzieht. Die ersten großen, auch literarisch bedeutenden deutschen Autobiographien im 18. Jahrhundert, Heinrich Stillings Jugend, Jünglingsjahre, Wanderschaft 
und häusliches Leben (1777-1804) und Karl Philipp Moritz، Anton Reiser (1785-90), sind deutlich von solchen Impulsen gespeist.

Die Grundlage für die rechte Bewältigung des Lebens ist die Bildung in der Kindheit und Jugend, die die Verwirklichung des geistigen und seelischen Potentials ermöglicht. Diese Ausbildung der im Menschen angelegten Kräfte wurde um 1800 zum Credo der Intellektuellen, das in Goethe und Wilhelm von Humboldt seine beredtesten Repräsentanten fand und auch den typisch deutschen Bildungsroman hervorbrachte. Es ist sicher kein Zufall, daß Goethe der Verfasser des ersten großen Bildungsromans und zugleich der einer umfangreichen Autobiographie ist, die ein Jahrhundert lang Ideal und Vorbild des Genres blieb.

In dem Unterschied zwischen aristokratischem und bürgerlichem Selbstverständnis liegt auch der zwischen Memoiren und Autobiographien begründet. Jene legen den Schwerpunkt der Selbstdarstellung in das öffentliche Leben des Menschen, seine soziale Einbettung, sein Wirken im Dienst von Krone und Staat, wie es auf monumentale Weise der Herzog von Saint Simon in der Zeit Ludwigs XIV. in Frankreich getan hat. Das Persönliche, Intime, der Umgang des Individuums mit sich selbst bleibt darin als irrelevant im Hintergrund. Es sind aber gerade diese Züge, die in der bürgerlichen Autobiographie ins Zentrum rücken, denn hier geht es eher um die Entfaltung des Ichs zur Persönlichkeit, sein Erfassen und Ergreifen der Welt und den Dialog mit der eigenen Seele. Daß gerade die Deutschen mit ihren Traditionen der Verinnerlichung diesen Typ von Selbstdarstellung immer besonders geschätzt haben, liegt auf der Hand. Die Prägung des Menschen wird dabei ebenso wichtig wie sein dann folgendes Wirken, und folgerichtig spielen dabei die Darstellung von Kindheit und Jugend, über die die Memoiren eher hinweghuschen, eine immer größere Rolle und nehmen immer größeren Raum ein. Goethes Autobiographie Dichtung und Wahrheit endet mit Mitte 20, und die populärste Selbstdarstellung des späteren 19. Jahrhunderts, Wilhelm von Kügelgens 1870 erschienene Jugenderinnerungen eines alten Mannes, die generationenlang zu den Standardwerken des bürgerlichen Bücherschranks gehörten, enden schon mit dem 18. Lebensjahr und an der Schwelle zum Erwachsenwerden mit einem erschreckenden Erlebnis des jungen Mannes, der die nackte Leiche seines einem Raubmord zum Opfer gefallenen Vaters findet.

Zunehmend verselbständigt sich in solchen Erinnerungsbüchern auch die 1 . Lebensphase, so daß sich bei Fontane und einigen anderen Autoren des 19. Jahrhunderts die Schilderung der Kinderzeit zu einem selbständigen Werk ausgewachsen hat, das auf die Fortsetzung gar nicht mehr angewiesen ist. Auch das hat wohl Fontane dazu bewogen, Meine Kinderjahre einen »autobiographischen Roman « zu nennen: Es ist ein in sich geschlossenes Werk, und der Autor hat mit dem zweiten Teil seiner Erinnerungen, Von Zwanzig bis 
Dreißig (1898), dem letzten zu Lebzeiten des Dichters erschienenen Werk, auch nicht an den ersten angeschlossen, sondern die eigentlichen Jugendjahre ausgespart.

Was im 18. Jahrhundert als Ausdruck des neuerworbenen bürgerlichen Selbstverständnisses und Selbstbewußtseins begann und in Goethes Dichtung und Wahrheit zu einem frühen, nie übertroffenen Höhepunkt führte, wurde im 19. Jahrhundert zur Selbstverständlichkeit und häufig auch zur Veräußerlichung. Das autobiographische Rinnsal wuchs zu einem Strom von Lebenserinnerungen an, der zur Zeit des späten Fontane rauschend dahinfloß. Er selbst hat in einem Brief angemerkt, daß kurz vor und nach Veröffentlichung seiner Kindheitserlebnisse verschiedene andere Zeitgenossen ebenfalls autobiographisch auf den Plan traten:

Die Zeit steht im Zeichen der fluthenden Biographie; Lübke, Pietsch sind kaum überwunden und schon sind 5 andre Richmond's in the field: Brugsch, Hanslick, Keller, Roquette, ich. Und wahrscheinlich viele andre noch. Gott gebe seinen Segen. ${ }^{13}$

Die Zahl der Erinnerungen, Denkwürdigkeiten aus meinem Leben, Memoiren usw. war groß, und längst lebten auch die verschiedensten Typen autobiographischer Literatur nebeneinander. Dichter, Musiker und bildende Künstler, Ärzte, Militärs und Schauspieler, Philosophen, Politiker und Pädagogen, Naturwissenschaftler, Juristen und Kaufleute - darunter bemerkenswerterweise auch etliche Frauen - gaben öffentlich Rechenschaft ab über ihr Werden und Wirken, ihre Erlebnisse und Erfahrungen - eine Fülle von kulturgeschichtlichem Material zur Zeit, das heute oft nicht genug für das Verständnis des 19. Jahrhunderts genutzt wird. Zu Fontanes Zeit herrschte dabei eher die bürgerliche Erfolgsbiographie vor, in der man seine glorreiche Karriere dem Publikum vorführte: wie herrlich weit man es gebracht hatte! Von diesen gesellschaftlich selbstsicheren Autobiographien wollte sich Fontane mit der Darstellung seiner Kinderjahre bewußt abheben.

Auch wenn die in den Autobiographien des 19. Jahrhunderts geschilderte Kinderzeit meist nicht zur Idylle verklärt wurde, sondern etwa Armut und familiäres Unglück, aber auch beunruhigende, verstörende und erschreckende Erlebnisse einschließt - der Maler Ludwig Richter zum Beispiel schildert die Wirkung der grausamen, als Kind erlebten Schlachtenszenen in den Napoleonischen Kriegen mit ungeschminkter Eindringlichkeit, und Kügelgen berichtet von alptraumartigen Visionen, die ihn als Kind heimsuchten -, ist doch ihr 
Grundton heiter und beschaulich, galten die Kinderjahre als Zeit der Unschuld und des Weltvertrauens. Zwar gibt es auch bei Fontane »Allerlei Gewölk« so die Überschrift des 17. Kapitels -, aber auch er empfindet seine frühen Jahre als »ein wunderbar schönes Leben in dieser kleinen Stadt, dessen ich noch jetzt, wie meiner ganzen buntbewegten Kinderzeit, unter lebhafter Herzensbewegung gedenke«. Besonnte Vergangenheit, der Titel der 1924 erschienenen Lebenserinnerungen des pommerschen Arztes, Malers und Schriftstellers Karl Ludwig Schleich, der in den ersten Kapiteln seines Buches wie Fontane den Reiz der Landschaft an der Odermündung schildert, kann als Stichwort für das Genre im 19. Jahrhundert gelten. Es ist nur eine andere Formulierung für das, was Fontane am Ende von Meine Kinderjahre mit dem Satz umreißt: »Alles war Poesie.«

Fontane war sich darüber im klaren, daß er mit der Beschränkung auf ein paar Kindheitsjahre von dem gängigen Schema der Selbstdarstellung abwich und ein literarisches Risiko einging, denn - so schrieb er an Georg Friedlaender -, »ich weiche ganz von dem Ueblichen ab und erzähle nur Kleinkram. Meine Überzeugung, daß das das Richtige sei, ist unerschüttert, aber daneben bleibt doch die Frage, ob ich's im Maß richtig getroffen habe und wenn richtig getroffen, ob das Publikum Lust hat, meinen Standpunkt gelten zu lassen. ${ }^{14}$ Fontane wollte auf diese Weise dem eigenen $>$ Personenkult $<$ und dem Vorführen berühmter Zeitgenossen, die man gekannt hatte, entgehen - Schwächen, die in den Autobiographien seiner Zeit grassierten und über die er seiner Tochter mißbilligend schrieb: „Das Operiren mit Größen und sich selber dabei als kleine Größe im Auge haben, immer Kunst, immer Literatur, immer ein Professor, immer eine Berühmtheit, - das alles ist vom Uebel. ${ }^{15}$

In einer Kindheitsschilderung konnte Fontane einmal dieser Gefahr ausweichen, und er konnte zum anderen ein viel geschlosseneres Werk liefern, als es die Darstellung seiner ganzen Lebensgeschichte ermöglicht hätte. Mit einer Kürzung um ein Drittel, wie sie der Herausgeber der Deutschen Rundschau, Julius Rodenberg, bei einem eventuellen Vorabdruck verlangte, konnte sich Fontane deshalb nicht einverstanden erklären und verzichtete trotz der finanziellen Einbuße lieber ganz auf die Vorveröffentlichung (nur zwei einzelne Kapitel, das 13. und das 16., erschienen vor der Erstausgabe in Zeitschriften). Auch der Künstler in Fontane hat also wohl für die Beschränkung auf einen überschaubaren, weit zurückliegenden Abschnitt plädiert und dabei genau den zeitlichen Abstand gewählt, den Walter Scott als Ideal für den historischen Roman deklariert und den auch Fontane selbst bei seinen beiden im eigentlichen Sinn historischen Romanen Vor dem Sturm (1878) und Schach von Wuthenow (1882) recht genau eingehalten hat: »vor sechzig Jahren«. Das eigentlich scheint die Rechtfertigung des schon erwähnten Untertitels »autobiographischer Roman « zu sein. Es läßt sich nämlich beobachten, wie Fontane mit zunehmendem Alter mehr in seinen Äußerungen über seine eigenen 
Werke die "Mache « betont und bemüht ist, in der Kunstgerechtigkeit vorbildlich zu wirken. Auch bei Meine Kinderjahre hat er den Anspruch erhoben, das Buch "gibt, dabei bleibe ich, doch wenigstens einen Fingerzeig, wie man die Sache anzufangen hat«, ja, er nannte es geradezu »einen Versuch « - heute würde man sagen: ein Experiment - und sprach von der »neuen Art der Behandlung. $\ll^{16}$ Der Untertitel soll also nicht sagen, daß die Erinnerungen des alten Herrn unzuverlässig sind; es ist noch nicht einmal der Bemerkung im Vorwort zu trauen, daß er »nicht von einzelnen aus jener Zelt her vielleicht noch Lebenden auf die Echtheitsfrage interpelliert « werden wollte. Der Untertitel soll vielmehr darauf hinweisen, daß das Buch eine Komposition darstellt, die nach künstlerischen Gesichtspunkten gestaltet ist. Wie immer gehört es dabei zu den Formkriterien des alten Fontane, daß sie nicht ein starres Schema werden, sondern daß die Gestaltung sich hinter einer zwanglosen Oberfläche verbirgt.

Schon der erste Absatz erscheint wie die Erfüllung dieses Programms, denn das Buch beginnt nach Romanmanier in der 3. Person mit einer distanziert erzählten, zeitlich und räumlich präzise erfaßten Situation: der Ankunft eines Herrn und einer Dame in einer Kutsche. Aber schon wenige Zeilen später wird die fiktionale Fassade durchbrochen, indem der Autor zur 1. Person überwechselt und das Paar als seine eigenen Eltern identifiziert.

Fontanes Meine Kinderjahre ist also künstlerisch ein Buch von seltener Geschlossenheit. Es schildert, wie der Autor selbst es in einem Brief charakterisiert hat, »das alltägliche Leben einer kleinen baltischen Stadt aus dem ersten Drittel dieses Jahrhunderts und desgleichen das alltägliche Leben eines norddeutschen Jungen aus derselben Epoche. $\ll^{17}$ Beides, so glaubte Fontane, sei bisher noch nicht geschildert worden. Tatsächlich sind damit die beiden zentralen Elemente von Meine Kinderjahre bezeichnet: die Stadt und der Junge, der sie erlebt.

Der Zeltraum umfaßt so wenige Jahre, daß das geschilderte Kind sich nahezu gleichbleibt und auch Vater und Mutter und die anderen Gestalten - die Geschwister spielen auffälligerweise fast gar keine Rolle - sich in seinem Bewußtsein nicht verändern, obwohl Fontane Typisches und Chronologisches mischt. Der Junge führt wie jedes Kind in dieser Swinemünder Welt gewissermaßen 2 Leben: das als Erziehungsobjekt der Erwachsenen und als beobachtender Teilnehmer ihres Lebens und das in seinem eigenen Kinderreich, in dem andere und manchmal für die Erwachsenen ganz unbedeutende Handlungsorte wichtig sind, wie etwa die vielen Böden der Apotheke, die "Störtebekers Kul'" oder das Wasser.

Die Stadt, der nahezu ausschließliche Schauplatz des Buches, ist ein Ort mit einer überschaubaren Bevölkerung und einer unverwechselbaren Physiognomie, der mit seinen Menschen, seiner sozialen Schichtung, seinen Ausflugsstätten, auch seinen Kuriositäten und dem sensationellen Verbrechen 
Mohrs und vor allem dem vielgestaltigen Leben am Wasser lebendig geschildert wird. In dieser Liebe zum Kindheitsort ähnelt Fontanes Meine Kinderjahre Fritz Reuters Meine Vaterstadt Stavenhagen (1856), von der der Verfasser gegen Ende bemerkt, er habe sie "gleichsam als Individuum« behandelt. Genau dasselbe ließe sich mit gutem Recht auch von Fontanes Swinemünde sagen, und man kann die Autobiographie als Huldigung an die »an Schwindel und Bummel so überreich gesegnete (übrigens dadurch nur um so interessantere) kleine Stadt $^{18}$ lesen, die Fontane auf ganz typische Manier - kein Autor hat wohl so häufig die Formulierung »ja und nein« verwendet - »sehr häßlich und sehr hübsch « nennt. Kein Wunder, daß Fontane nach Erscheinen des Buches sagen konnte: "Den Swinemünder Weihnachtsmarkt habe ich literarisch beherrscht. ${ }^{19}$ Beides, Kindesleben und -erziehung und Kindheitsort, werden bewußt als historisch begriffen, als einem anderen Zeitalter zugehörig, das mit seinem Meißner Geschirr, seinen Schinkelschen Möbeln, seinen Kinderspielen und sogar seinen jetzt ausgestorbenen Menschentypen untergegangen ist. Fontane gibt dabei nicht nur ein Genrebild des Alltagslebens, sondern bezieht in einem eigenen Kapitel auch die »große Welt « mit ein, deren Echo auch Swinemünde und den Jungen erreicht: etwa den griechischen und den polnischen Freiheitskampf, die Pariser Julirevolution und die französische Eroberung von Algerien. Auch der Schatten Napoleons spukt wegen der Vorliebe Louis Henri Fontanes für die Generäle des Kaisers gerade in der Apothekersfamilie noch stark herum. Wieder läßt sich hier ein Zug erkennen, den der Sohn vom Vater geerbt hat: die Begeisterung für die menschlichen Elemente der Historie und für die spannenden geschichtlichen Ereignisse kurz, das balladeske Geschichtsverständnis, das bei Fontane literarisch so fruchtbar geworden ist.

Den Zeitgenossen Fontanes mußten seine Genrebilder tatsächlich wie aus einer anderen Welt erscheinen, denn wie hatten sich Deutschland und die Welt im Laufe des 19. Jahrhunderts verändert! Während Fontanes Swinemünder Kinderzeit lebte Goethe noch. Als er aber in den 90er Jahren Meine Kinderjahre schrieb, war das provinzielle Preußen der Biedermeierzeit, in der der junge Theodor noch die Herren aus dem 18. Jahrhundert erlebte, längst zum Zentrum des neuen deutschen Reiches, sein König der deutsche Kaiser geworden. Die Industrialisierung hatte die ökonomische und natürliche Landschaft völlig verändert und aus dem beschaulichen Berlin eine Riesenmetropole gemacht, in der der materielle Fortschritt, aber auch das soziale Elend mit Händen zu greifen waren. Der Sozialismus, den es um 1830 als politische Kraft noch gar nicht gab, hatte Bismarcks Sozialistengesetze unbeschadet überstanden und schickte sich an, die stärkste Partei im Reichstag zu werden, ja, Bismarck selbst war schon entlassen und der Erste Weltkrieg nur noch 20 Jahre entfernt. Der Aufstieg der Naturwissenschaften hatte das gesamte Denken und religiöse Empfinden verändert und unerhörte Neuerungen mit sich 
gebracht. In den 90er Jahren gab es schon elektrisches Licht und die ersten Kraftfahrzeuge; und auf Fontanes eigenem Fachgebiet, der Pharmazie, hatte sich die rapide Entwicklung der Chemie revolutionierend ausgewirkt. Die Apotheken verkauften zunehmend mehr chemische Mixturen statt Kräutermischungen. Und Fontane selbst? Aus dem Jungen, in dem sich das poetische Gemüt geregt hatte, war nach langen Entbehrungen, Niederlagen und Umwegen der bedeutendste deutsche Romancier seiner Zeit geworden, dessen erster Roman erst in seinem 59. Lebensjahr erschienen war.

In jeder Hinsicht lag die Beschaulichkeit des kleinen Ostseestädtchens im 1. Drittel des Jahrhunderts weit zurück und mußte die Zeitgenossen des alten Fontane wie aus einer anderen Zeit berühren. Fontane selbst fand den Zauber seiner Kindheit schon 30 Jahre später nicht mehr wieder. Als er 1863 Swinemünde zum erstenmal seit seinem Abschied als 12jähriger wiedersah, berichtete er seiner Frau in einem Brief, wie sich alles verändert hatte:

Um 4 waren wir in Swinemünde.

An der Stelle, wo ich (es war ein wackliges altes Fachwerkhaus, drin die Ressource war) als 14jähriger Junge, angetan mit einem blauen Bastard von Frack und Jacke, getanzt und bei »Pfänderspiel« und »Wohnungsvermieten « zuerst die Unbefriedigtheit des jungen Poetenherzens empfunden hatte, erhebt sich jetzt ein großes Hotel mit vielen Balkonen und einem Eckturm, ein Gasthaus, das in Erscheinung und Größe keinem Berliner etwas nachgibt. Auch das Geschlecht der Kellner schien aus Berlin zu sein, womit ihr Urteil gesprochen ist. Ich zog mich um und ging dann in die Stadt. Es ist alles anders geworden. Leutnants und Soldaten treiben sich in den Straßen herum, dazwischen Marineoffiziere und Matrosen »von der Flotte«, ein riesiger Leuchtturm flankiert und überragt das ganze Bild, Dampfer kommen und gehn, und zu beiden Seiten des Stroms erheben sich die neuen Befestigungswerke, mit ihren Türmen und Bastionen. All das ist neu. Aber auch die Stadt selbst hat sich sehr verändert, und in abermals 30 Jahren wird sie den Charakter einer kleinen Schifferstadt mit Giebelhäusern völlig verloren haben. Diese Giebel, die Bäume vor den Türen und eine Art Gitter, das hürdenartig diese Bäume einschloß, waren das hübscheste an der Stadt, aber alles das ist auf dem Punkt zu verschwinden. Nur der Kastanienbaum steht noch, aus dessen Spitze ich (beim Kastanienpflücken) niederstürzte, wobei einer der untenstehenden Jungens ausrief: »Donnerwetter, nu kommt ne groBe.«

Dies führt mich natürlich auf das Haus, drin ich 5 Jahre lang gelebt, gelernt, gespielt, gelacht, geweint habe. Es ist total runtergekommen. Die Apotheke ist verlegt (Notiz für Mama: da, wo Schlächter Fietzkers Haus 
stand), und in dem Lokal, wo sonst rezeptiert wurde und wo der katholische Gehülfe dem protestantischen Kollegen mit dem Messingleuchter einen Schlag auf den Kopf gab, ist jetzt ein schmieriger Kaufmannsladen. Der Flur, die Küche, die winklige Treppe, die Einteilung der Zimmer ist (wenigstens an der linken, d. h. an der Wohnseite) unverändert geblieben, aber wiewohl es nie was Schönes war, so hat es sich doch bedeutend verschlechtert; denn alles ist dreckig und absolut ruppig geworden. Die Hof- und Garteneinrichtung ist total verändert, weil man nämlich aus dem ehemaligen Apothekenbesitz zwei Häuser gemacht hat; was früher das Nebenhaus war, für Materialkammer, Remise, Hühner- und Pferdestall, ist jetzt ein selbständiges Haus geworden, zu dem der ehemalige Hof jetzt noch als Hof gehört. Da nun dadurch das eigentliche Apothekenhaus ohne Hof sein würde, so hat der jetzige Besitzer aus dem, was früher Garten war, einen schmuddligen Hof gemacht, auf dem Heringsfässer liegen und der parallel mit dem Vorderhause (ganz so, wie es in Letschin war), an dem ehemaligen Kramerschen Gartenzaun entlang einige schlechte Nebengebäude aufweist. Nur der Nußbaum steht noch, der damals seine noch jungen Zweige in das Fenster von Papas Stube, da, wo sein Sekretär mit der ewig knarrenden Klappe stand, hineinwachsen ließ. Ich bin in allen solchen Stücken so unsentimental wie möglich, und ich kann nicht sagen, daß das alles mich tief ergriffen hätte; aber von leiser Wehmut, von einer gewissen Herbststimmung wird das Herz doch beschlichen.

\section{Dunkle Zypressen - \\ Ring dich nicht $\mathrm{ab}$. \\ Es wird doch alles vergessen -}

so ähnlich sagt Storm, und er hat recht. Immer wieder lief ich durch die Straßen der Stadt, aber ich sah kein bekanntes Gesicht, sie sind alle fort, verzogen, die meisten sehr weit.

Der alte Hofrat Kind lebt noch, aber ich hatte nicht den Mut, ihn aufzusuchen. ${ }^{20}$

Der Zauber Swinemündes war dahin ... Doch nein, er lebt in Meine Kinderjahre, ja, es gibt wenige andere deutsche Kleinstädte aus dem frühen 19. Jahrhundert, deren Bild lebendiger geblieben ist als gerade das von Fontanes Swinemünde. 
Anmerkungen

${ }^{1}$ Karl Kraus, Die Fackel 15 (30.5.1913) Nr. 376/377, S. 21; 15

(19.9.1913) Nr. 381/382/383, S. 73.

${ }^{2}$ Der Zuschauer 2 (Jan.-Jun. 1894) S. 225; Westermanns Monatsbefte 39 (1895) Bd. 77, S. 263.

${ }^{3}$ Brief an Georg Friedlaender, 9. 5. 1892 (HB 4.194).

${ }^{4}$ Brief an Carl Robert Lessing, 15. 6. 1892 (HB 4.199).

${ }^{5}$ Die Zitate stammen der Reihenfolge nach aus folgenden Briefen: an Carl Robert Lessing, 23. 5. 1892 (HB 4.198), an Wilhelm Hertz, 30. 5. 1892 (HB 4.198), an Carl Robert Lessing, 15. 6. 1892 (HB 4.200), an dens., 18. 6. 1892 (HB 4.201), an Friedrich Stephany, 9. 8. 1892 (HB 4.203), an dens., 31. 8. 1892 (HB 4.209).

${ }^{6}$ Brief an Karl Zöllner, 8. 8. 1892 (HB 4.202).

${ }^{7} \mathrm{HB} 4.227$.

${ }^{8}$ Tagebuch 1892 (DuD 121).

${ }^{9}$ An eine Dame, 22. 6. 1895 (HB 4.454).

${ }^{10} \mathrm{P}$. J. Anderson, ") Meine Kinderjahre $<$ die Brücke zwischen Leben und Kunst. Eine Analyse der Fontaneschen Mehrdeutigkeit als Versteck-Sprachspiel im Sinne Wittgensteins « in: Fontane aus heutiger Sicht. Analysen und Interpretationen seines Werkes, hg. von Hugo Aust. München 1980, S. 143182.

${ }^{11}$ Justinus Kerner, Das Bilderbuch aus meiner Knabenzeit. Erinnerungen aus den Jahren 1786-1804, in: J. K., Ausgewählte Werke, Stuttgart 1981, Vorrede, S. $113 \mathrm{f}$.

${ }^{12} Z$ it. nach: Ekkhard Verchau, Theodor Fontane. Individuum und Gesellschaft, Frankfurt a. M./Berlin/Wien 1983 (Fontane Bibliothek, Ullstein Taschenbuch, 4604), S. 106.

${ }^{13}$ An Wilhelm Hertz, 3. 12. 1893 (HB 4.313). - Wilhelm Lübke (1826-93, Kunsthistoriker), Lebenserinnerungen (1891) - Ludwig Pietsch (1824-1911, Publizist und Zeichner), Wie ich Schriftsteller geworden bin, 2 Bde. (1893/94) - Heinrich Karl Brugsch (1827-94, Archäologe), Mein Leben und mein Wandern (1894) - Eduard Hanslick (1825-1904, Musikkritiker), Aus meinem Leben, 2 Bde. (1894) - Gottfried Keller (1819-90), Gottfried Kellers Leben. Seine Briefe und Tagebücher, hg. von Jakob Bächtold, 3 Bde. (1892-96) - Otto Roquette (1824-96, Literaturhistoriker und Schriftsteller), Siebzig Jahre. Geschichte meines Lebens, 2 Bde. (1894).

${ }^{14}$ 26. 12. 1892 (HB 4.243).

${ }^{15}$ 9. 7.1893 (HB 4.267).

${ }^{16}$ An Martha Fontane, 9. 7. 1893 (HB 4.267), und an Julius Rodenberg, 1. 12. 1893 (HB 4.312) 
${ }^{17}$ Brief an Siegfried Samosch, 15. 1. 1894 (HB 4.320).

${ }^{18}$ Brief an Otto Schöneberg, 24. 11. 1892 (HB 4.236).

${ }^{19}$ Brief an Hermann Scherenberg, 2. 1. 1894 (HB 4.318).

${ }^{20}$ 24. 8. 1863 (HB 2.104-106). 